\title{
Using Noldus Observer XT for research on deaf signers learning to read: An innovative methodology
}

\author{
Daphne A. Ducharme And Isabelle ArCand \\ University of Ottawa, Ottawa, Ontario, Canada
}

\begin{abstract}
Despite years of research on the reading problems of deaf students, we still do not know how deaf signers who read well actually crack the code of print. How connections are made between sign language and written language is still an open question. In this article, we show how the Noldus Observer XT software can be used to conduct an in-depth analysis of the online behavior of deaf readers. First, we examine factors that may have an impact on reading behavior. Then, we describe how we videotaped teachers with their deaf student signers of langue des signes québécoise during a reading task, how we conducted a recall activity to better understand the students' reading behavior, and how we used this innovative software to analyze the taped footage. Finally, we discuss the contribution this type of research can have on the future reading behavior of deaf students.
\end{abstract}

Despite years of research on the reading problems of deaf students, we still do not know how deaf signers who read well actually crack the code of print (Allen \& Schoem, 1997). What we do know is that sign language and written language have very different structures (Mayer \& Akamatsu, 2003), which makes direct connections between the two difficult (Chamberlain \& Mayberry, 2000). Nevertheless, some deaf signers do learn to read well. How connections are made between sign language and written language is still an open question.

Previous research has established correlations between signing ability and reading ability, as well as between speaking ability and reading ability. However, we have yet to learn more about the online behavior of deaf readers. The purpose of this article is to show how we were able, with the Noldus Observer XT software, to take an in-depth look at the online behavior of deaf readers in a time-effective manner.

We will first examine factors that may have an impact on reading behavior. Then, we will outline the methodology used to analyze online behavior. Finally, we will discuss the contribution that this type of research can have on the future reading behavior of deaf students.

\section{Factors That May Have an Impact on Reading Behavior}

Three factors have recently been discussed in the literature as having an impact on the reading outcomes of deaf readers. First, the use of specific signing skills - namely, fingerspelling, initializing, and chaining - has been suggested as a way to create associations between sign and print. Second, it has been suggested that deaf teachers and hearing teachers make different use of these skills.
Third, students who are taught by deaf and hearing teachers seem to also use different reading strategies that reflect the mode of teaching to which they are exposed. These three factors will now be examined in more detail.

Signing skills. Many authors (e.g., Hirsh-Pasek, 1987; Padden \& Ramsey, 1998) have suggested that the success of deaf readers is due to their ability to create associations between two systems: sign language and written language. Hearing readers read aloud in order to generate associations between the oral and written modalities of the same language; deaf readers use fingerspelling and initializing to generate associations between sign and written language.

Fingerspelling consists of producing a word by making various manual codes that correspond to letters of the alphabet, thereby allowing the signer to manually construct a written word. The initializing technique is similar to fingerspelling, but its representation is very limited. In initializing, only the first letter of the oral translation of the signed word to be communicated is produced. Both these techniques are often used by deaf people to communicate a noun or a word that is part of their vocabulary but for which no specific sign exists (Hirsh-Pasek, 1987; Musselman, 2000). Research regarding the role of fingerspelling and initializing for children learning to read is still lacking (Padden \& Ramsey, 1998).

A third technique for creating associations between oral and signed languages is called chaining (Padden \& Ramsey, 1998). Chaining is a technique used by certain teachers in order to help the child make associations between the signed word, the written word, and the fingerspelling of that same word. When using this technique, the teacher can produce a sign, which is then followed by the finger-

D. A. Ducharme, daphne.ducharme@uottawa.ca 
spelling of the same word; alternatively, she can write a word, point to the written word, and then repeat the fingerspelling of the word. In their research, Padden and Ramsey (1998) found that chaining is used more frequently in residential schools for deaf children than in public schools, regardless of the teacher's hearing status. Padden and Ramsey (2000) wrote that the deaf teachers they observed made greater use of chaining than did the hearing teachers. Their hypothesis with regard to that observation was that deaf teachers use this technique more frequently because of their intuitive knowledge of the necessity to understand the relations between signing, fingerspelling, and writing. There are limited data about the role of chaining in deaf children's learning of reading (Andrews \& Mason, 1991; Padden \& Ramsey, 2000). Further research is needed to better understand how deaf children decode written text and whether this technique is useful in learning to read, as well as how all of the signing skills discussed in this section can help deaf students learn to read.

Different use of signing skills by deaf and hearing teachers. As previously outlined, a second factor may have an impact on deaf students' reading outcomes - that is, the different uses deaf teachers and hearing teachers make of signing skills in American Sign Language (ASL). Padden and Ramsey (1998) examined the use of fingerspelling and initializing by hearing and deaf teachers in public and residential schools. Their study yielded important findings: Deaf teachers used fingerspelling twice as often as did hearing teachers; and teachers who used fingerspelling often also tended, several times within the same segment, to repeat the words that were manually spelled. Also, deaf teachers frequently used fingerspelling in chaining. According to Padden and Ramsey (2000), deaf teachers were more inclined to create connections among words written on the board, their corresponding signs, and the fingerspelling of those same words while they taught, using chaining to switch from one representation to another.

One hypothesis about deaf and hearing teachers' differing uses of communication skills (such as chaining, fingerspelling, and initialized signs) may have to do with the level of knowledge and fluency of ASL. It is believed that deaf teachers use these skills because they are an integral part of everyday ASL communication. But it could be that these skills instead reflect the individual teacher's personal communication style (Padden \& Ramsey, 2000). However, this cannot be entirely true; Ramsey (1997) examined the teaching approaches of deaf and hearing teachers and found a deaf teacher in a public school who used fingerspelling and chaining less often than did a hearing teacher in a residential school. Ramsey also observed a group of children who were taught by a hearing and a deaf teacher in an environment where ASL was the main teaching language. The children were prompted to compare English to ASL, to reflect on language, and to learn how language could be used as a communication tool. Also, to promote learning, interaction was encouraged. Ramsey uncovered yet another factor - teaching environment- that seemed to influence the use of skills by teachers. We still need to know more about how the skills used by a teacher can influence a student's learning, and, perhaps, looking more closely at online behavior can add to our knowledge base about teacher and student strategies with respect to teaching and learning to read.

\section{A Student's Reading: The Mirror Image of His or Her Teacher's Approach?}

Padden and Ramsey (1998) examined the links that were present between teacher behavior and student behavior when a text was being read. They suggested that a student can be influenced by the reading strategies used by his or her teacher. In their study, Padden and Ramsey (1998) observed the reading strategies used by the student when he or she had to read a text and then retell the story. The teacher was present and could give clues to the student when needed. Afterward, the researchers compared the student's interpretation of the text to the story itself. Fingerspelling and mouthings, as well as Signing Exact English and ASL, were observed. The student's comprehension level was measured by analyzing his or her interpretation of the text.

Two reading approaches were identified from Padden and Ramsey's (1998) observations. One was a word attack and analysis approach; the other was a meaning-making approach, which was characterized by a search for the global meaning of the story. In the word attack and analysis approach, reading becomes a matching task in which each written word is individually translated in sign. Attention is focused on words and how they are constructed, rather than on the overall meaning of the sentence. In the meaningmaking approach, the deaf reader looks at the written words at first and then looks beyond them to extract the larger meaning of the text. Typically, the student does a preliminary reading activity to browse through the pages of the text before he or she starts to sign. In this approach, the student is not constrained by sentence endings, punctuation marks, or words that he or she cannot decode. The student tries to find a meaning beyond the sentence or the paragraph, even if he or she is unable to decode every single word.

In addition to identifying the two approaches outlined above, Padden and Ramsey (1998) observed that students mirrored their teacher's approach. That is, students whose teacher used a word attack approach also favored such an approach to reading, and, conversely, if their teacher favored a meaning-making approach, so too did the students.

The research conducted thus far on reading strategies in deaf children and their teachers has been rather time consuming, owing to the lack of online techniques or equipment available to conduct the in-depth analyses required. The Noldus Observer XT is a welcome innovation in this respect.

Prior research has explored and established correlations between signing ability and reading ability, as well as between speaking ability and reading ability (Chamberlain \& Mayberry, 2000; Hirsh-Pasek, 1987; Leybaert, 1993; Musselman, 2000; Padden \& Ramsey, 1998, 2000). Although these findings provide valuable information about factors that influence reading, we are particularly interested in studies that examine online reading behav- 
ior. Findings from such studies may be useful for improving teaching methods by showing, in a minute-by-minute analysis of reading behavior, what exactly is happening during reading.

The research described above does address the issue of online reading behavior to some degree; however, it has been conducted on English and ASL. Accordingly, an important question to investigate is whether these results can be replicated with French and langue des signes québécoise (LSQ). LSQ is the signed language used by deaf signers who are descendants of French Canadians and live in parts of Canada, such as Quebec, Ontario, and New Brunswick. LSQ signers generally learn to read French, whereas ASL signers learn to read English. Just as French and English share similarities, such as a common alphabet, ASL and LSQ share handshapes; however, they are distinct languages with different lexical, grammatical, and pragmatic features. One notable difference is that native signers of LSQ typically rely less on fingerspelling and use more mouthings than do ASL users. Therefore, it is pertinent to examine whether results obtained with ASL can be transferred to LSQ. On the basis of the previously outlined research, we propose the following questions, with respect to deaf LSQ signers learning to read French:

1. What is the online behavior of profoundly deaf LSQ signers when reading?

2. Does student strategy use differ according to level?

3. Do teachers' strategy uses differ?

\section{THE NOLDUS OBSERVER XT}

The Observer XT is a behavioral coding and analysis software that supports all the steps of a research project like ours: coding scheme development, data entry, data management, and data analysis, as well as interrater reliability analysis. Once the hardware is set up by the provider, video data can be collected with the system or imported from another source. In the case of our study, we recorded video off site and imported our footage into the system. We then designed a coding scheme comprising labels for the strategies that were used by the teachers and students we had videotaped. The Observer XT software made it possible for us to keep all of our video events in one database and gave us access to all of that data at all times. Each video was separately visualized and coded for behaviors (i.e., strategies).

The data management feature of the software was particularly useful. The Observer XT organizes coding schemes, observational data, independent variables, data filters, and analysis results. The independent variables are used for labeling treatment, subject ID, observer, and any other important circumstances. Another useful feature of the system is the availability of all of the data in the project at all times. This means that one can extract only the bits of each video that are relevant to each research question. Filters can also be built on the basis of combinations of independent variables, behaviors, and time criteria. The software also makes possible the analysis of large groups of observations at once, saving a tremendous amount of time.

Using video analysis with the Noldus system, we conducted a study bringing together our three research questions in order to go beyond just establishing correlations between reading ability and signing ability. Using the Noldus Observer XT software, we analyzed the actual online behavior of deaf LSQ-signing children and their tutor by videotaping them during a reading task.

\section{Method}

Participants. Case studies were conducted with 3 adolescent males who were profoundly deaf from birth. Their primary language was LSQ, and their written mode of communication was French. Two tutors also participated in the study. The first tutor was hearing, had a native competence in French, and had a near-native level of signing competence. She was the participants' French teacher. All 3 students had already read some texts with her in their French class: They were, therefore, familiar with her teaching approach. The second tutor was deaf, had a native competence in LSQ, and had a near-native competence in written French. She taught at the same school, but none of the 3 students had been in any of her classes. The students were not familiar with her approach, but they knew her, having seen her many times in the halls and at school functions. The choice of both a hearing and a deaf tutor reflected our expectation that signing behavior and teaching practices might differ as a result of language background, as demonstrated by Padden and Ramsey $(1998,2000)$.

The participants' French reading ability was measured with two tests: the reading comprehension subtest of the French version of the Canadian Achievement Test (the Test de rendement pour francophones; Sarrazin, 1996) and the French version of the Peabody Picture Vocabulary Test known as Échelle de vocabulaire en images Peabody (Dunn, Thériault-Whalen, \& Dunn, 1993). On the basis of the results of the tests, we categorized the 3 participants as weak, low-average, and average readers. A demographic questionnaire also yielded information regarding age of sign-language acquisition, language of parents (deaf or hearing), language of siblings, use of LSQ, and reading behavior.

Procedure. The participants were asked to read and retell a narrative text with the help of a tutor. They were videotaped reading the story, signing "aloud" (with assistance if needed), and then retelling it (with prompts if needed). Each reading activity lasted approximately $30 \mathrm{~min}$. Stimulated recall sessions (Ericsson \& Simon, 1993) were then conducted by a trained research assistant within 3 days of the reading activity, with the assistance of an LSQ/French interpreter. Each of these sessions lasted approximately $60 \mathrm{~min}$.

The reliability and validity of stimulated recall as a source of data have long been supported by Ericsson and Simon (1993). In their research, these authors examined verbal reports in order to determine how they could be used to inform studies of cognitive processes. They showed that verbally communicating ideas does not affect one's cognitive processes and that one can report on the contents of his or her short-term memory. They concluded that a verbal report is as valid and reliable as any other type of information.

In our study, stimulated recall involved conducting an interview with the teacher or student while watching the videotape of the reading and retelling activity he or she had participated in. The student and/or teacher was encouraged to comment on the reading activity process and to provide insight about how the student was able to achieve an understanding of the text with or without help. The interviews focused on what specific LSQ skills (i.e., fingerspelling, initialized signs, or chaining) and what reading strategies (seeking meaning or word attack) were used by the teacher and student to help them understand the text. These sessions were videotaped for later analysis with the Noldus Observer XT system.

Six dyads were videotaped: weak reader with hearing teacher $(\mathrm{W}+\mathrm{H})$, low-average reader with hearing teacher $(\mathrm{L}+\mathrm{H})$, average reader with hearing teacher $(\mathrm{A}+\mathrm{H})$, weak reader with deaf teacher 
$(\mathrm{W}+\mathrm{D})$, low-average reader with deaf teacher $(\mathrm{L}+\mathrm{D})$, and average reader with deaf teacher $(A+D)$. Each of the 3 participants interacted with both tutors on separate occasions, reading a different text (of similar type and level of difficulty) each time. This allowed us to compare the strategies used by all 3 signers/readers in constructing meaning from text. An additional comparison was obtained from observing each of the 3 students interacting with both types of tutors.

In addition to videotaping the reading activity, we videotaped the stimulated recall activity. The number of interviews for this activity increased to 12 because we interviewed the teacher and the student separately as each watched the same reading activity. We separately interviewed the weak reader and the hearing teacher from the $\mathrm{W}+\mathrm{H}$ reading dyad, the low-average reader and the hearing teacher from the $\mathrm{L}+\mathrm{H}$ reading dyad, the average reader and the hearing teacher from the $\mathrm{A}+\mathrm{H}$ reading dyad, the weak reader and the deaf teacher from the $\mathrm{W}+\mathrm{D}$ reading dyad, the low-average reader and the deaf teacher from the $\mathrm{L}+\mathrm{D}$ reading dyad, and the average reader and the deaf teacher from the $\mathrm{A}+\mathrm{D}$ reading dyad.

It was expected that (1) the better reader would use a meaningmaking strategy for comprehending the text; (2) the weaker reader would favor a word-attack strategy; (3) the deaf tutor would make more use of fingerspelling, initialized signs, and chaining than the hearing tutor; and (4) the weaker reader would achieve better understanding of the text when reading with the deaf tutor.

\section{Results}

The amount of footage generated from the reading and recall activities totaled $18 \mathrm{~h}$. Examining this type of foot- age to identify types of strategies can be very time consuming. In order to make comparisons and answer our research questions, we turned to Noldus. All of the video footage was analyzed using the Noldus Observer XT software. The story-reading videos and the stimulated recall videos were first converted into computerized files. The stimulated recall videos with the student and those with the teacher were then coded according to the strategies used in constructing the meaning of the text. Variables observed for each type of strategy included frequency and efficacy (i.e., did it work?). We were then able to compare the strategies used by each teacher (deaf and hearing) with the reading strategies of all 3 students. A second comparison among all 3 students sought to identify differences between the better and the weaker reader in the study. These comparisons were facilitated by the data selection feature of the Observer XT software, which made all of the data available to us at once. Furthermore, we were able to create a filter for selecting different combinations of independent variables - such as reading level and teacherand behaviors, such as strategies.

The use of video analyses and, especially, the Noldus Observer XT software allowed us to gain a sense of each teacher's style of teaching through an in-depth analysis of the types and frequency of the strategies used with all students, regardless of the student's profile. This analysis

Table 1

List of All Strategies, as Generated From the Videotaped Data

\begin{tabular}{|c|c|}
\hline Behavior Name & Description \\
\hline Drawing & The student or teacher uses a drawing to construct meaning. \\
\hline Use of context & $\begin{array}{l}\text { The student or teacher uses information around the word (in the sentence or in the picture he con- } \\
\text { structs of the story). }\end{array}$ \\
\hline Analogy & Find similarity in the meaning of two words. \\
\hline Background knowledge & Use of background knowledge, such as memories, experience, knowledge \\
\hline Reference to pictures in the story & The student points to or mentions the pictures shown in a text to construct its meaning. \\
\hline Global meaning & The student uses the whole text to gain a general understanding of the story or a paragraph. \\
\hline Grammatical structure & Use of grammatical features of French or LSQ to construct the meaning of a sentence \\
\hline Writing & $\begin{array}{l}\text { The teacher writes out one or more words to help the student better understand or distinguish a dif- } \\
\text { ficult word. }\end{array}$ \\
\hline Fingerspelling & The teacher manually spells a words using signs for each letter. \\
\hline Role-playing & The student or teacher takes on the role of a character to act out the story. \\
\hline Lipreading & $\begin{array}{l}\text { The student or teacher uses lip movements to pronounce a word as a way of priming its } \\
\text { comprehension. }\end{array}$ \\
\hline Logic & The student or teacher uses reasoning or coherence in the text to help find the meaning of a word. \\
\hline Words from same family & The student or teacher uses a word with a similar root to help understand a difficult word. \\
\hline Answer in LSQ & $\begin{array}{l}\text { The teacher shows the student the sign in LSQ, knowing that he knows the concept and will under- } \\
\text { stand the word. }\end{array}$ \\
\hline Synonyms & $\begin{array}{l}\text { The student or teacher uses another word that has an equivalent meaning to help understand the prob- } \\
\text { lem word. }\end{array}$ \\
\hline Mime & The student or teacher uses gestures to illustrate an action. \\
\hline Reference to past personal experience & Reference to past experience \\
\hline Student creates a picture in his mind & $\begin{array}{l}\text { The student or teacher mentions that the student creates a picture in his mind linked with what is hap- } \\
\text { pening in the text. }\end{array}$ \\
\hline Explanation in LSQ (teacher) & $\begin{array}{l}\text { The teacher gives the student information in LSQ to help him understand a new concept; the student } \\
\text { does not know the word or the sign for this new concept. }\end{array}$ \\
\hline Reference to LSQ & The student or teacher uses rules of syntax or morphology in LSQ to construct meaning. \\
\hline Vocabulary learning & Strategies to help the student learn new vocabulary \\
\hline Independent search for meaning & The student tries to construct meaning on his own. \\
\hline Teacher guides the student & The teacher gives an example, a clue, or an answer to help the student construct meaning. \\
\hline Phonological or visual decoding & The student or teacher uses letter-sound correspondences or the visual aspect of a word. \\
\hline Questioning & The teacher asks the student questions, back and forth, until the student understands. \\
\hline DNP & Use of the Dynamique naturelle de la parole as an intervention method \\
\hline Comprehension check & The teacher questions the student to check his comprehension of a sentence, a paragraph, or the story. \\
\hline
\end{tabular}


Table 2

Excerpt of One Video Event Analysis

\begin{tabular}{|c|c|c|c|c|c|}
\hline Event & Event Start Time & Participant(s) & $\begin{array}{c}\text { Participant } \\
\text { Modifier }\end{array}$ & Behavior & $\begin{array}{l}\text { Behavior } \\
\text { Modifier }\end{array}$ \\
\hline 1 & $+0: 06: 27.000$ & HearingTeach & & Teacher guides the student & effective \\
\hline 3 & $+0: 08: 08.266$ & HearingTeach & & Teacher guides the student & effective \\
\hline 4 & $+0: 08: 08.266$ & Student\&HearingTeach & & Independent search for meaning & effective \\
\hline 5 & $+0: 09: 31.266$ & Student\&HearingTeach & & Reference to LSQ & unclear outcome \\
\hline 8 & $+0: 12: 34.900$ & Student\&HearingTeach & & Reference to LSQ & unclear outcome \\
\hline 9 & $+0: 12: 34.900$ & HearingTeach & & Teacher guides the student & effective \\
\hline 10 & $+0: 12: 44.000$ & HearingTeach & & Teacher guides the student & effective \\
\hline 11 & $+0: 12: 44.000$ & HearingTeach & & Explanation in LSQ (teacher) & effective \\
\hline 12 & $+0: 12: 44.000$ & Student\&HearingTeach & alone & Independent search for meaning & effective \\
\hline 17 & $+0: 13: 46.500$ & Student\&HearingTeach & & Global meaning & unclear outcome \\
\hline 18 & $+0: 13: 46.500$ & Student\&HearingTeach & alone & Student creates a picture in his mind & unclear outcome \\
\hline 19 & $+0: 14: 21.000$ & HearingTeach & & Teacher guides the student & effective \\
\hline 20 & $+0: 14: 21.000$ & HearingTeach & & Synonyms & effective \\
\hline 21 & $+0: 15: 09.000$ & HearingTeach & & Synonyms & effective \\
\hline 22 & $+0: 15: 09.000$ & HearingTeach & & Teacher guides the student & effective \\
\hline 23 & $+0: 16: 08.000$ & Student\&HearingTeach & & Context & effective \\
\hline 24 & $+0: 16: 08.000$ & Student\&HearingTeach & alone & Independent search for meaning & effective \\
\hline 25 & $+0: 16: 40.000$ & Student\&HearingTeach & alone & Independent search for meaning & effective \\
\hline 26 & $+0: 16: 40.000$ & Student\&HearingTeach & & Context & effective \\
\hline 27 & $+0: 17: 50.001$ & HearingTeach & & Global meaning & effective \\
\hline
\end{tabular}

yielded a list of strategies, like the one shown in Table 1. Table 2 shows the analysis of one event in our database. This analysis was constructed while we viewed the videotaped event and the table side by side on the computer screen. We coded the behaviors into the table while watching the tape, with the time codes automatically recorded for the start and finish of the behavior exhibited.

Once a video event has been coded from beginning to end, the data management feature of the software becomes particularly relevant. This feature allows us to call up all videoclips in the database that have been coded as representative of this strategy in all events. We can then verify that all of those clips are effectively exemplars of the same strategy, across all events, and with all participants. The data management feature offers multiple ways of viewing the data, including the option of viewing all strategies used by one teacher with one or many students, or viewing the strategies used by different teachers with the same student, and so on.

With the data management feature, we were able to effectively categorize the types of strategies used by the hearing teacher and by the deaf teacher (Research Question 3). This led to the observation of differences between them. For example, the deaf teacher relied more on meaningmaking strategies, whereas the hearing teacher used more word-attack strategies. It was also possible to reanalyze the data with a focus on the students; this led to the finding that the students used different strategies with each teacher, seemingly adapting to the style of the teacher.
This led to a detailed list of all of the strategies used by the deaf LSQ signers in our study (Question 1), such as the list shown in Table 1. We also observed differences among students of different reading levels (Question 2).

Finally, from the lists of behaviors (i.e., types of strategies) generated with the Noldus software, we were able to easily create Excel graphs. For example, Figures 1 and 2 illustrate the strategy use of the deaf teacher and of the hearing teacher, as well as the frequency and efficacy of each strategy used. These graphs, as well as others created from the database, gave us a visual profile of each teacher's overall strategy use and of each student's overall strategy use, as well as between-teachers and betweenstudents comparisons.

\section{DISCUSSION}

The study described in this article has shown how online behavior can be captured on videotape and analyzed in a way that can generate results that inform researchers on the frequency and efficiency of strategies for teaching reading to deaf students. Previous research of the same nature - that is, on online behavior - has proven to be very time consuming (Ducharme \& Hurst, in press). Although it was previously possible to use software to facilitate this type of research, it was not possible to integrate data capture, coding, analysis, and management of many videos in one database with the same ease offered by the Noldus Observer XT software. We believe this may be one 
Strategies Used by the Deaf Teacher

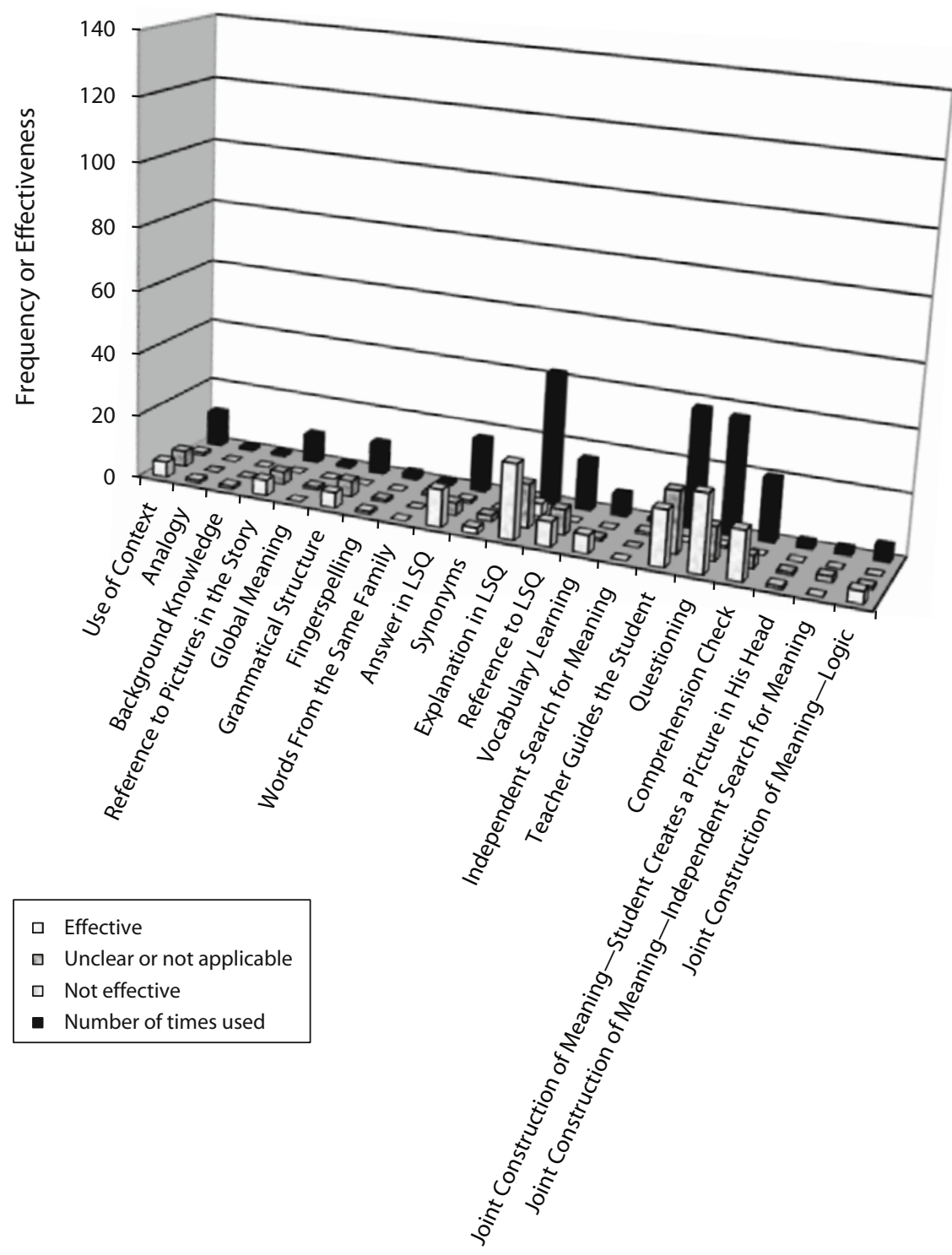

Type of Strategy Used

Figure 1. Overall strategy use by the deaf teacher, including frequency of usage and effectiveness, in an Excel table derived from the Noldus Observer XT database.

reason that many other researchers have chosen to conduct correlation studies instead of in-depth analyses of online teacher and/or student behavior.

As we have shown, Noldus Observer XT software can be used effectively for analyzing online data, particularly those of videotaped participants using sign language. We were able to use this tool to generate, from human behavior, lists of types of behavior - namely, strategies for reading. These identified strategies, generated from observations of individual participants, were then compared with strategies used by other participants - both students and teachers. A number of within-participants and between- participants analyses were facilitated and performed. From these analyses, profiles of behavior were drawn and illustrated using Excel.

The data thus transformed can tell a story in a much more eloquent way, enabling teachers to draw conclusions about what works with students. For example, one can see the teaching strategies used by the hearing teacher and the deaf teacher, and such visualization can promote exchanges of teaching practices.

With respect to within-participants comparisons, we can see from the data that the more advanced reader is more efficient in his strategy use than are the less skilled 


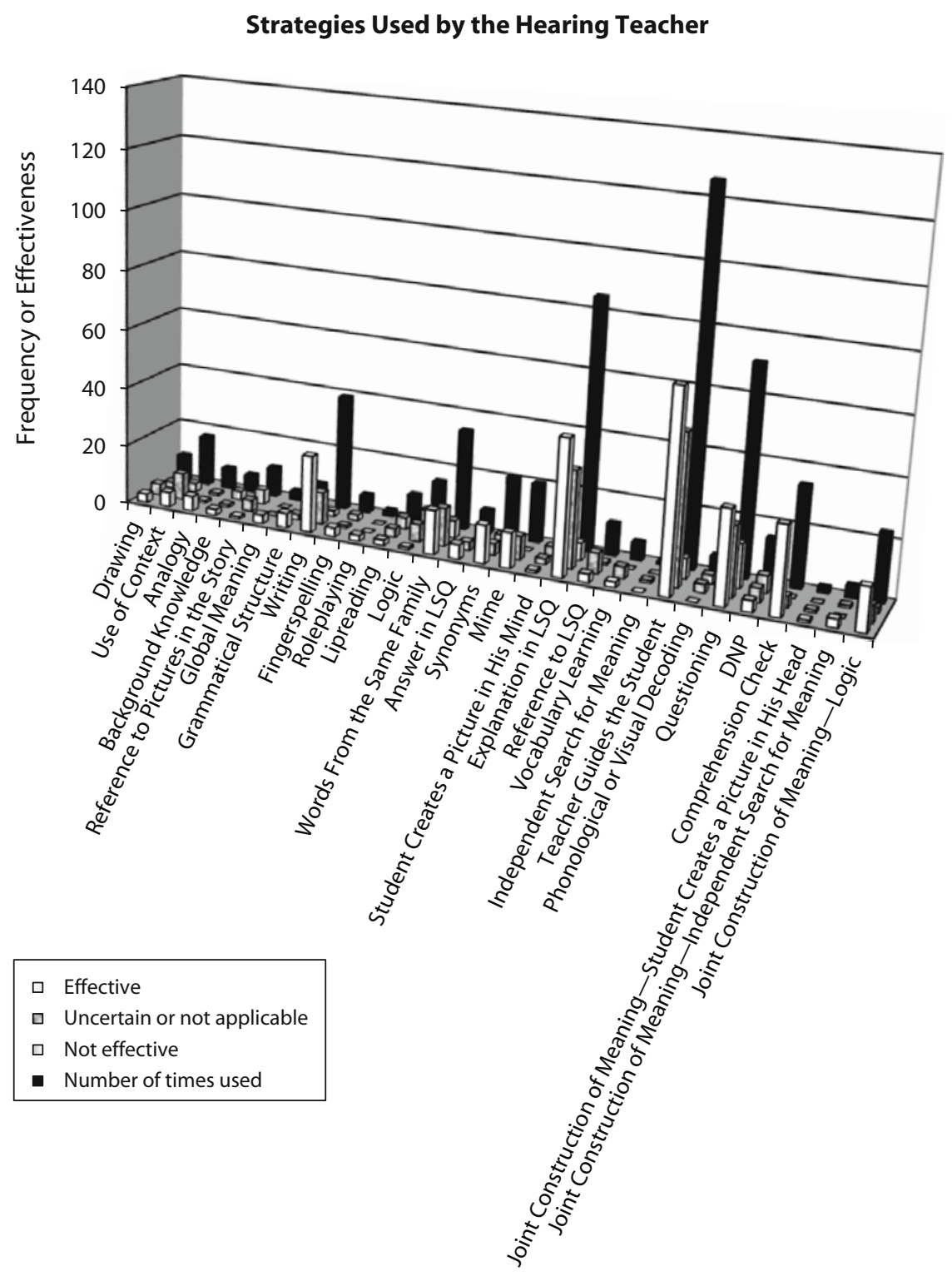

Type of Strategy Used

Figure 2. Overall strategy use by the hearing teacher, including frequency of usage and effectiveness, in an Excel table derived from the Noldus Observer XT database.

readers in our study. Such a finding may provide to teachers clues about which strategies to promote in order to bring weaker readers to a higher level of skill.

These are only a few examples of the benefits of indepth analysis of online behavior. Using the Noldus Observer XT to analyze videotaped sequences of teaching and learning provides unlimited opportunities for examining and reexamining data and for multiple comparisons.

\section{Conclusion}

One long-standing preoccupation of educators of the deaf has been to help their students learn to read. Previous research has tackled the problem in a variety of ways, with limited success. In this article, we have shown another way of exploring how deaf readers learn to read: by studying their online reading behavior. The innovative nature of the Noldus Observer XT software - a powerful tool for studying videotaped data - provides new hope for this type of research. By learning more about the online behavior of deaf readers (i.e., their reading processes), we can inform teachers about better teaching methods. We believe these methods will, in turn, lead to better learning and, ultimately, improve reading among deaf students.

The case-study nature of our research limits the generalizations that can be made from the results. It is not possible to know, for example, whether the differences 
observed were just between these 2 teachers or are representative of other hearing and deaf teachers. A further study with replicates of both pupils and teachers would enable such conclusions to be drawn. We have shown that such a study is possible, less time consuming, and more effective than was the case before the existence of the Noldus Observer XT software. We hope this will encourage further research of a similar nature.

\section{AUTHOR NOTE}

Portions of this article appeared previously in "Using Noldus' The Observer XT to Analyze Videotaped Footage of Deaf Signers Learning to Read With a Tutor," by D. A. Ducharme and I. Arcand, 2008, in A. J. Spink, M. R. Ballintijn, N. D. Bogers, F. Grieco, L. W. S. Loijens, L. P. J. J. Noldus, et al. (Eds.), Proceedings of Measuring Behavior 2008. Copyright 2008 by Noldus Information Technology. Used with permission of the author. This research was funded by Grant 410-2004-2033 of the Social Sciences and Humanities Research Council of Canada. Correspondence concerning this article should be addressed to D. A. Ducharme, School of Rehabilitation Sciences, Faculty of Health Sciences, University of Ottawa, 451 Smyth Rd., K1H 8M5, Ottawa, Canada (e-mail: daphne.ducharme@uottawa.ca).

\section{REFERENCES}

Allen, T. E., \& Schoem, S. R. (1997, May). Educating deaf and hardof-hearing youth: What works best? Paper presented at the Combined Otolaryngological Spring Meetings of the American Academy of Otolaryngology, Scottsdale, AZ.

ANDREWS, J. F., \& MASON, J. M. (1991). Strategy usage among deaf and hearing readers. Exceptional Children, 57, 536-545.

Chamberlain, C., \& MaYberry, R. I. (2000). Theorizing about the relationship between American Sign Language and reading. In C. Cham- berlain, J. P. Morford, \& R. I. Mayberry (Eds.), Language acquisition by eye (pp. 221-259). Mahwah, NJ: Erlbaum.

Ducharme, D. A., \& Hurst, L. (in press). Être sourd et apprendre à lire: Une étude de cas des stratégies de lecture d'un élève de 14 ans. Revue du Nouvel Ontario.

Dunn, L. M., Thériault-Whalen, C. M., \& Dunn, M. L. (1993). Échelle de vocabulaire en images Peabody. Toronto: Psycan.

Ericsson, K. A., \& Simon, H. A. (1993). Protocol analysis: Verbal reports as data. Cambridge, MA: MIT Press.

HIRSH-PASEK, K. (1987). The metalinguistics of fingerspelling: An alternate way to increase reading vocabulary in congenitally deaf readers. Reading Research Quarterly, 22, 455-474.

LeybaerT, J. (1993). Reading in the deaf: The roles of phonological codes. In M. Marschark \& M. D. Clark (Eds.), Psychological perspectives on deafness (pp. 269-311). Hillsdale, NJ: Erlbaum.

Mayer, C., \& AKamatsu, C. T. (2003). Bilingualism and literacy. In M. Marschark \& P. E. Spencer (Eds.), Oxford handbook of deaf studies, language, and education (pp. 136-147). New York: Oxford University Press.

Musselman, C. (2000). How do children who can't hear learn to read an alphabetic script? A review of the literature on reading and deafness. Journal of Deaf Studies \& Deaf Education, 5, 9-31.

Padden, C., \& Ramsey, C. (1998). Reading ability in signing deaf children. Topics in Language Disorders, 18, 30-46.

Padden, C., \& Ramsey, C. (2000). American Sign Language and reading ability in deaf children. In C. Chamberlain, J. P. Morford, \& R. I. Mayberry (Eds.), Language acquisition by eye (pp. 165-189). Mahwah, NJ: Erlbaum.

RAMSEy, C. L. (1997). Deaf children in public schools: Placement, context, and consequences. Washington, DC: Gallaudet University Press.

SARRAZIN, G. (1996). Test de rendement pour francophones. Toronto: Psychological Corp.

(Manuscript received October 30, 2008; revision accepted for publication March 5, 2009.) 